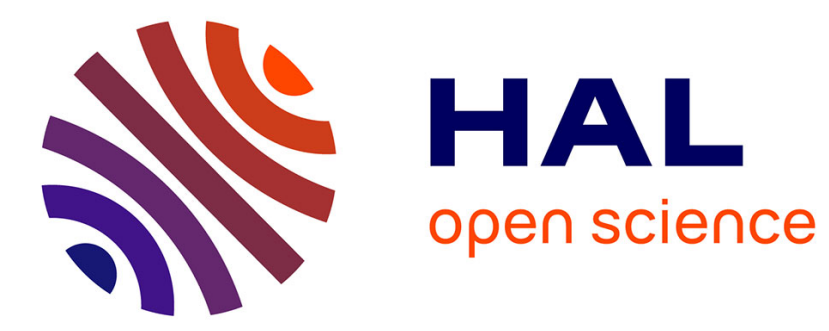

\title{
Expatriation et notabilité. L'évergétisme et la diaspora iranienne
}

Fariba Adelkhah

\section{To cite this version:}

Fariba Adelkhah. Expatriation et notabilité. L'évergétisme et la diaspora iranienne. Politix, 2004, 17 (65), pp.73-92. 10.3406/polix.2004.1610 . hal-01019924

\section{HAL Id: hal-01019924 \\ https://hal-sciencespo.archives-ouvertes.fr/hal-01019924}

Submitted on 7 Jul 2014

HAL is a multi-disciplinary open access archive for the deposit and dissemination of scientific research documents, whether they are published or not. The documents may come from teaching and research institutions in France or abroad, or from public or private research centers.
L'archive ouverte pluridisciplinaire HAL, est destinée au dépôt et à la diffusion de documents scientifiques de niveau recherche, publiés ou non, émanant des établissements d'enseignement et de recherche français ou étrangers, des laboratoires publics ou privés. 


\section{Expatriation et notabilité. L'évergétisme et la diaspora iranienne} In: Politix. Vol. 17, N65. Premier trimestre 2004. pp. 73-92.

Citer ce document / Cite this document :

Adelkhah Fariba. Expatriation et notabilité. L'évergétisme et la diaspora iranienne. In: Politix. Vol. 17, N²5. Premier trimestre 2004. pp. 73-92.

doi : $10.3406 /$ polix.2004.1610

http://www.persee.fr/web/revues/home/prescript/article/polix_0295-2319_2004_num_17_65_1610 


\section{Résumé}

Expatriation et notabilité. L'évergétisme dans la diaspora iranienne

\section{Fariba Adelkhah}

La figure notabiliaire est omniprésente dans la société politique iranienne. C'est notamment elle qui articule au pouvoir d'Etat la diversité régionale, ethnique ou religieuse, dans un pays multiculturel, multilinguistique, et multiconfessionnel. Aussi la continuité de la figure notabiliaire de l'Empire à la République l'emporte sur la discontinuité. Dans les provinces, le rôle joué par les hommes de confiance, notamment lors des élections, est considérable. En même temps, l'acte de se porter candidat est une façon de se voir reconnaître le statut de notable, en dépit éventuellement d'une chance minime d'être élu(e). Toutefois la notabilité n'a pas de frontière, que celle-ci soit sociale ou géographique, et l'on peut devenir notable dans l'émigration dont l'aventure fournit souvent elle-même des ressources spécifiques en la matière. Ainsi le marché cambiaire a représenté depuis 1980 une ressource notabiliaire formidable pour les émigrés, compte tenu de la faiblesse et de la dépréciation continue du rial des années 1980 jusqu'à 2001. Les investissements évergétiques que prodigue l'« homme de bien » de la diaspora sont ainsi devenus spectaculaires. Ce travail analyse le parcours évergétique de l'homme de bien, d'une part, comme une facette de la recomposition de la communauté iranienne dans la diaspora et, de l'autre, comme une interrogation décalée sur l'une des institutions les plus anciennes de la société d'origine, le vaqf.

\section{Abstract \\ Notable and Exile. Figures of Eminence as a Social and Religious Dynamics in Iranian Society Fariba Adelkhah}

The eminent man is omnipresent in Iranian political Society. It is he in particular who forms the link between state power and the regional, ethnic and religious diversity in a multicultural, multilingual and multiconfessional country. The continuity of the eminent man from the Empire to the Islamic Republic also defies the discontinuity of this transition. In the provinces the role played by such men, particularly at the time of elections, is considerable. At the same time, the act of running for office is a way of being recognised as a notable or as an eminent man (motamed), despite perhaps the minimal chance of being elected. However, eminence has no border and can not be reduced nor to a given social group neither to a particular geographical frame, and one can become eminent in exile where the adventure often provides specifie resources for becoming notable. Thus, the currency market has represented since 1980 a significant resource for exiles, taking in to account the continuous weakness and depreciation of the rial between 1980 and 2001. The investments which the Diaspora's notables lavish on their beneficiaries have thus become truly spectacular. This work analyses the notables' beneficence activities, on the one hand, as a facet of the recomposition of the Iranian community in diasporas and, on the other, as a shifted inquiry into one of the most ancient institutions of their homeland, the vaqf.

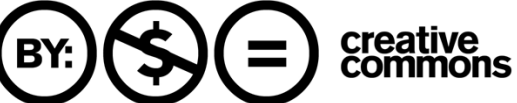




\title{
Expatriation et notabilité
}

\author{
L'évergétisme dans la diaspora iranienne
}

Fariba ADELKIIAI I

$\mathrm{L}$

a figure notabiliaire est omniprésente dans la société politique iranienne. C'est notamment elle qui articule au pouvoir d'Etat la diversité régionale, ethnique ou religieuse, dans un pays multiculturel, multilinguistique et multiconfessionnel. En dépit de son autoritarisme, le régime du Shah reposait sur de telles interactions entre le local et le national et sur l'influence sociale ou économique des "mille familles ${ }^{1}$ ». La Révolution de 1979 semble avoir détrôné nombre d'entre elles et en a couronné d'autres en inaugurant de nouvelles ressources notabiliaires, comme par exemple les états de service révolutionnaires, la participation à l'effort de guerre (éventuellement par martyrs interposés) ou la légitimité religieuse et cléricale. Mais, à y bien regarder, la continuité de la figure notabiliaire de l'Empire à la République l'emporte sur la discontinuité. D'une part, l'islam et le nationalisme étaient déjà des éléments de respectabilité sociale avant 1979. D'autre part, la Révolution, sous couvert de populisme et de célébration des " déshérités ", a été largement faite par et autour des notables, en particulier dans les provinces. Même les élites sociales qui ont été ébranlées par la création de la République islamique n'ont pas tardé à regagner au moins une partie de leur ascendant sur la scène locale ou nationale, par exemple à l'occasion des élections législatives et, depuis 2000, municipales. 
Dans les provinces, les commerçants, les propriétaires de puits ou de terres, les autorités cléricales, voire les intellectuels, sont des "personnalités $\mathrm{d}^{\prime}$ éminence ${ }^{2}$ » qui s'efforcent généralement d'occuper le terrain politique. Soit ils se présentent eux-mêmes aux diverses élections, soit ils participent aux campagnes des candidats et à la sélection des candidatures en tant que "grands électeurs" - ou plus exactement en tant qu' «hommes de confiance" (mo'tamed), désignés par le gouverneur au vu de la reconnaissance sociale dont ils jouissent et chargés d'établir l'acceptabilité des candidatures avant le transfert de leurs dossiers au Conseil de surveillance de la constitution. Réciproquement, l'acte de candidature est une façon de se voir reconnaître le statut de notable, hormis même toute chance d'être élu(e). Certain(e)s sollicitent l'agrément du Conseil de surveillance comme stratégie d'ascension socioprofessionnelle ou de respectabilité. Tant et si bien que tout un débat a lieu depuis plusieurs années sur le rejet des candidatures par ce même Conseil, les uns s'indignant que ce dernier porte atteinte à l'honorabilité des personnes mises en cause, les autres - en particulier les autorités politiques - assurant que le refus d'un candidat ne compromet pas l'intégrité et la foi des intéressés ${ }^{3}$.

L'une des lignes de continuité de la figure notabiliaire en Iran est l'ethos du javânmard - littéralement $l^{\prime}$ « homme intègre " - et les pratiques de don dont il s'accompagne. La plupart des personnalités, politiques ou autres, de la République islamique agissent ou sont perçues dans les termes de ce style de $v_{i e}^{4}$. La principale innovation sociale récente, de ce point de vue, est que désormais des femmes se posent en évergètes, et donc en notables, ce qui eût été impensable il y a une vingtaine d'années, sinon à titre d'exception. L'on disait ainsi que la dynastie des Qadjar (1796-1925) n'avait connu qu'un seul « homme » (étant entendu que les seuls vrais mâles sont les javânmard): la princesse Fakhroddoleh, célèbre pour ses dons à des œuvres sociales et religieuses. Une autre ligne de continuité tient à l'institution sociale du vaqf (bien de mainmorte) qui, en Iran comme dans les autres pays musulmans, est partie intégrante de la vie quotidienne. L'on peut ainsi créer un vaqf pour dédommager les croyants dont on aurait volé les chaussures au seuil de la mosquée, ou les servantes qui auraient cassé leur cruche. Néanmoins, il est clair que le vaqf est particulièrement utile au notable en mal de constitution d'une réputation et d'une clientèle, d'autant plus qu'historiquement il a

2. Mines (M.), Public Faces, Private Voices. Community and Individuality in South India, Berkeley, University of California Press, 1994.

3. Adelkhah (F.), Etre moderne en Iran, Paris, Karthala, 1998 ; Adelkhah (F.), «Iran: femmes en mouvement, mouvement de femmes ", in Bennani-Chraibi (M.), Fillieule (O.), dir., Résistances et protestations dans les sociétés musulmanes, Paris, Presses de Sciences Po, 2003. Rappelons que le Conseil de surveillance de la Constitution est le garant de la conformité des lois de la République avec les principes islamiques et constitutionnels. A ce titre, il exerce un contrôle sur les candidatures aux diverses élections.

4. Adelkhah (F.), Etre moderne en Iran, op. cit., chap. 1 et 2. 
toujours permis de mettre son capital évergétique à l'abri de la concupiscence de l'Etat, de toute autre forme de menace ou, tout simplement, de la suspicion sociale. Le don, comme pratique centrale de la notabilité, ne doit pas prêter à confusion. Certes, il est un facteur d'interaction entre les différentes couches sociales, et donc d'intégration. Mais il est simultanément un élément de distinction et de pouvoir. Dans le velours de sa bienfaisance, l'évergète exerce son autorité d'une main de fer, tant dans sa famille que dans sa circonscription d'influence. En un mot il est un homme de la hiérarchie sociale.

Mais reste à savoir comment cette figure de la notabilité affronte l'épreuve de l'émigration en tant qu'expérience de marginalité sociale. Même si ceux qui partent sont loin d'être les plus déshérités (contrairement à l'idée reçue), ils affrontent la déchirure de la séparation - parfois dans des conditions très dures lorsqu'ils fuient la répression politique, la conscription, ou tout simplement la justice et le fisc. Le caractère épique des récits auxquels donne lieu l'expatriation est généralement proportionnel à la trivialité et à la souffrance du voyage et des conditions d'installation concrètes dans un pays étranger. Bien sûr, la diaspora iranienne qui s'est constituée depuis une vingtaine d'années et qui serait maintenant forte de quelque trois millions d'âmes, est socialement diversifiée. Les dits taghouti (idolâtres) de l'ancien régime qui se sont exilés après la Révolution (ou à son approche) et se sont pour l'essentiel réfugiés en Californie nagent pour la plupart dans l'opulence. Il en est de même des grands commerçants de Dubaï, de quelques marchands de tapis établis dans les capitales européennes ou à Tokyo, etc. Mais la crise économique a provoqué le départ de personnes provenant de milieux moins favorisés ou, plus récemment, des classes moyennes (l'Iran semble par exemple connaître une accélération de la fuite de ses cerveaux). Dans tous les cas il y a quelque chose de pathétique dans l'exil, que cette misère soit celle des conditions de vie et de travail, celle de l'ennui et de la nostalgie que l'on traîne dans le printemps éternel de Los Angeles, ou encore celle de l'impossibilité de rattraper le temps enfui de sa jeunesse. Il n'empêche que l'on peut devenir notable dans l'émigration et que cette figure sociale s'inscrit désormais dans la dimension transnationale. C'est ce que nous allons essayer de montrer, à partir d'enquêtes de terrain que nous avons effectuées depuis 1998, successivement, dans les Emirats arabes unis et le sultanat d'Oman, en Californie et à Tokyo, en soulignant, d'une part, la singularité de la stratégie notabiliaire sur le lieu de l'expatriation et, d'autre part, l'écho en retour de cette figure notabiliaire dans son terroir d'origine. 


\section{Comment on devient notable dans l'émigration}

La première particularité du phénomène notabiliaire dans l'expatriation est son rapport à l'espace local dont il provient. En Iran, même l'évergète qui s'affirme dans son village, son quartier, sa ville ou sa province n'éprouve guère le besoin de mettre en scène ce cadre qui lui est naturel. Il se contente de le dominer de sa bienfaisance. En revanche, le notable de la diaspora entretient avec son terroir d'origine un rapport fantasmatique. Il ne cesse de l'exalter et de s'y référer, à l'image de ce propriétaire d'un restaurant de Dubaï qui y a mis en évidence quatre horloges donnant gravement l'heure de Londres, de Téhéran, de New York et de Grash, une ville de 25000 habitants du sud de l'Iran dont il est originaire. Dans des "circuits de migration 5 » très fluides où la mobilité d'un lieu d'expatriation à l'autre est la règle, l'ancrage à son lieu de naissance semble être une nécessité vitale. Véritable manifeste du transnationalisme marchand, l'entretien que nous avons eu avec T., commerçant d'origine khorramshahri, chiite, basé à Dubaï depuis quatorze ans, est de ce point de vue révélateur. Sa femme et ses quatre enfants résident au Canada depuis un an, pour des raisons de scolarité. Son père est resté dans la "ville ensanglantée " (Khorramshahr, entièrement détruite lors de la guerre avec l'Irak). Pour notre interlocuteur, au commencement est l'obligation éthique de gagner sa vie pour assurer celle des siens : "Comme le dit le Prophète, on peut travailler n'importe où et avec n'importe qui [sous-entendu : les non-musulmans], là où il y a du pain et où l'on peut ainsi servir l'islam. " Le premier devoir est de satisfaire les besoins de la "petite famille", puis, si cela est possible, ceux de "la grande famille » (ce qui peut désigner l'ensemble des " musulmans » et les " déshérités »). "Je suis venu ici pour travailler. Si je ne travaille pas je dois $\mathrm{m}^{\prime}$ en aller. Nous n'avons pas de problème de religion ni de nation ", précise T., pour ajouter aussitôt : "Selon le poète irakien Sadoun Jaber, celui qui perd de l'or le récupère un jour ou l'autre, mais celui qui perd sa patrie, où pourra-t-il la retrouver? Tout le monde est lié à son lieu de naissance. L'important est de protéger sa lignée et son authenticité. "

Cette relation privilégiée au lieu de naissance, à la " petite patrie " n'est pas exclusive de la nostalgie et de l'attachement que l'on éprouve pour la nation. Il y a d'ailleurs dans le vocabulaire une ambivalence, puisque le terme vatan renvoie étymologiquement aussi bien au lieu de naissance, en arabe, et à la mère patrie, en persan ou en turc. Un Iranien, selon qu'il est arabophone, persanophone ou turcophone, accordera au mot une connotation différente même si en arabe la notion de vatan se réfère également à la patrie au sens

5. Rouse (R.), "Mexican Migration and the Social Space of Postmodernism ", Diaspora, 1 (1), 1991, p. 14. Cf. également Hsu (M.Y.), Dreaming of Gold, Dreaming of Home. Transnationalism and Migration between the United States and South China. 1982-1943, Stanford, Stanford University Press, 2000. 
politique du terme. Mais, pour ce qui nous retient ici, c'est bien le rapport au local qui fournit les principales ressources de la stratégie notabiliaire dans l'expatriation. L'évergète de la diaspora, en faisant acte de générosité, contribue à délimiter une communauté d'originaires qui se tisse progressivement dans les lieux de culte, dans les "nappes " (c'est-à-dire les repas) offertes à l'occasion d'une célébration religieuse, ou dans diverses activités culturelles. Par exemple, à Dubaï, des personnes influentes et fortunées, originaires des villes de Grash et de Lar, ont conjugué leurs efforts pour ouvrir deux hosseiniyeh (lieux de culte dédiés à l'imam Hossein) de part et d'autre de la Creek. Y sont célébrés pour l'essentiel les deuils et la commémoration de Moharram (le deuil lié à la bataille de Kerbela) ainsi que celle de Ramadan. Mais ces bâtiments peuvent aussi accueillir des réunions associatives non religieuses. Dans tous les cas leur fréquentation - et bien sûr leur financement - sont des moments de distinction sociale qui permettent aux notables de se poser en " hommes de confiance » vis-à-vis d'une communauté en perpétuel renouvellement du fait de la circulation des migrants.

A Tokyo, l'on dénombre trois lieux de culte : une mosquée dans l'enceinte de l'ambassade de la république islamique d'Iran, un hosseinieh créé par M. Mardani (un marchand de tapis d'une grande notoriété) et un mahdavieh (lieu de culte dédié au douzième imam), dans lesquels sont distribués des repas iraniens - souvent le seul de la semaine, donc particulièrement prisé et où se tiennent diverses réunions, notamment de célébration des morts (dont la fréquentation au moins hebdomadaire est régulière). Ce à quoi il faut ajouter pas moins de quatre guildes de marchands de tapis et deux revues: Pol-e Abrisham ("Le pont de la soie ») et Roshanai ( La lumière »). Cette fragmentation des institutions de la colonie iranienne de Tokyo est d'autant plus frappante que cette dernière ne comporte plus que quelques milliers de personnes depuis l'entrée en crise de l'économie japonaise. Elle suffit à suggérer que les stratégies notabiliaires sont des sites de compétition sociale ou économique entre aspirants à la notabilité, autant que de fabrication de lien social entre les expatriés. Plus précisément, le lien social procède de la compétition sociale et de la guerre des évergètes. L'ethos de la "bonne action", en même temps qu'il se réclame de la solidarité et qu'il fabrique cette dernière, enregistre et engendre de la hiérarchie, du pouvoir et donc des conflits - fussent-ils feutrés - entre le donateur et les donataires.

L'aventure de l'émigration fournit souvent elle-même des ressources notabiliaires spécifiques. Cela est patent dans le cas des monarchies arabes du Golfe qui, à l'instar des Emirats arabes unis ou du sultanat d'Oman, pratiquent le système des sponsors pour octroyer des visas, des titres de séjour de plus longue durée, voire des opportunités de commerce ou d'investissement. Un tel système offre l'occasion à certaines personnes, d'origine étrangère ou naturalisées depuis plusieurs générations, de devenir 
des gate-keepers. Tel est notamment le cas de familles baloutches ou originaires de la côte iranienne méridionale qui se sont installées dans les ports de la péninsule arabique dès le $X I X^{e}$ ou le début du $X X^{e}$ siècle. Leur rôle d'intermédiation administrative ou économico-financière les prédispose à se constituer un capital social de relations et de clientèles.

Dans le contexte complètement différent des Etats-Unis, les dispositions légales et fiscales des organisations caritatives non lucratives (charities) ont également facilité les stratégies notabiliaires. L'appropriation du champ religieux à Los Angeles et à Irvine se réalise surtout par le biais des pratiques évergétiques. Le don reste au cœur de la sociabilité de la communauté. Les troncs destinés à recueillir l'obole des passants sont disposés dans la plupart des commerces iraniens : épiceries, librairies, disquaires, restaurants. Ils sont régulièrement alimentés. De façon plus institutionnalisée, les autorités et les centres religieux perçoivent les impôts islamiques. Quant aux grands évergètes, ils se livrent, à l'américaine, au fund raising ou, comme disent les nostalgiques du bazar, au golrizoun (littéralement « jeter des fleurs»), voire à la pratique de ventes aux enchères au bénéfice, qui de la construction d'un lieu d'accueil pour le troisième âge ou d'un centre religieux, qui des mutilés, qui des aveugles, etc. La modalité la plus courante en est un repas de gala, avec ou sans musique, assorti de l'allocution d'une personnalité ou d'une vedette. En soi cet évergétisme n'a rien d'exclusivement islamique, même s'il correspond à un devoir religieux. Il semblerait plutôt que ce soient les Iraniens juifs qui aient, les premiers, développé ce modèle, lequel a par la suite essaimé dans les autres composantes de la communauté iranienne - la pratique du don étant, de pair avec la religiosité, un élément de convergence et de retrouvailles interconfessionnelles.

La transplantation en Californie du répertoire du javânmard et de la bonne action (nikoukâri) est ainsi l'un des principaux agents de l'invention conservatrice de l'«iranité ». Elle permet tout à la fois l'affirmation des particularismes confessionnels ou ethniques (ou encore des choix politiques) et leur dépassement. On doit notamment souligner combien des personnalités connues pour leurs sentiments laïques ou actifs dans le monde $\mathrm{du}$ " show-biz » sont loin d'être insensibles à la magie de la bonne action et des vœux. Le public, très « branché » et huppé, du film La couleur de Dieu, de Majid Majidi, projeté à Beverly Hills et à Passadena en juin 2000, se montrait particulièrement généreux à l'égard de la Fondation de l'enfant (bonyad-e koudak) qui recueillait ses oboles à la sortie de la séance. Les vedettes iraniennes de Beverly Hills sont réputées ne pas être les dernières à faire preuve de comportements chevaleresques ou de générosité publique pratiques qui semblent bien être les moyens par lesquels le monde de la 
scène accède à la légitimitét . Et l'éditeur des Yellowv Pages de San Diego, Mirdavood Rezai, préface ainsi sa livraison de l'an 2000 :

"Je remercie Dieu tout puissant, le Créateur, de m'avoir accordé, à moi le moindre de ses esclaves, le don de vous servir. C'est donc en vous offrant pour la cinquième année consécutive cet annuaire professionnel de poche que je remplis ma dette morale $(d e y n)$ à l'étranger. Elle consiste à établir de la solidarité et des liens entre les Iraniens, à élever le nom plein de fierté de l'Iran, à protéger les convictions justes, la croyance en Dieu et aux prophètes. J'ai toujours sollicité auprès de Dieu la grâce, durant mon hégire [l'exil, en référence au départ du Prophète à Médine] obligé, d'avoir un style et une action dignes des émigrés (moliâdjer) des Livres saints pour ne pas avoir honte lors de mon retour dans le pays de mes ancêtres et pour être fidèle au serment que j'ai fait au moment de mon départ. "

Le style du javânmardi est omniprésent dans la communauté. C'est cette qualité que l'éditorialiste de l'hebdomadaire Teluran International, Shahabod Noori, attendait de la célèbre chanteuse de variété Googoosh lorsque celle-ci put enfin quitter l'Iran pour se produire en Amérique du Nord: "Bienvenue, Mme Googoosh! Nous avons entendu que vous allez bien gagner votre vie au cours de ce voyage. Nous l'espérons. Si tel est le cas, nous vous prions de bien vouloir créer un centre de bienfaisance qui portera votre nom et se fixera pour objectif d'aider les Iraniens ou les Afghans, ou les artistes isolés, voire oubliés et sans soutien, ou encore les petites orphelines afin d'immortaliser votre nom. Pour ce faire il suffit de 500000 dollars, ou un peu plus ${ }^{7}$. " C'est aussi à cette aune que sont appréciés les autres ou qu'est jugée la République islamique : sur les ondes de la Radio Sedâ-ye Iran, le régime honni est accusé d'être nâ-javânmard (" non jacianmard »), alors que Sadate est présenté comme s'étant comporté en jaônnmard en accueillant son ami le Shah d'Iran contraint à l'exil ${ }^{8}$. Enfin, dans l'imaginaire de la communauté, chaque mort qui se respecte a été un vrai javânmard. C'est par exemple le cas d'une certaine Sepideh Namazikhah, décédée à l'âge de 17 ans d'une longue maladie, dont l'abnégation, réelle ou supposée, a inspiré à ses proches la création d'une fondation portant son nom et vouée à des ceuvres éducatives. Il ne s'agit nullement d'un cas isolé, car à l'origine d'un bon nombre des fondations caritatives créées dans les différentes villes en Iran, à l'initiative de la diaspora, se trouve un mort muni de cette qualité sin' qua non de l'être parfait. La commémoration des défunts qui, comme en Iran, est un rite majeur et régulier de la vie sociale, se

6. On a par exemple parlé du jờînmardi de Shahram, un chanteur qui avait annulé son concert pour ne pas pénaliser un collègue qui, au même moment, devait se trouver sur scène dans la méme localité; ou encore du don religieux d'un autre chanteur ayant engagé le savoir culinaire de sa mère afin de servir du gheimeh polo à ses amis (Tehran International, 179, 28 avril 2000, p. 68).

7. Tilum Intirnational, 188, 30 juin 2000, p. 7.

8. Khandaniha, 528, 1378/1999, p. 40. 
confond avec l'exaltation de leur générosité. L'observation des cérémonies dans les différents centres et lieux de réunion islamique le confirme d'emblée. Et c'est sur le même ton qu'une famille irano-juive rendra hommage aux bonnes actions (nikoukâr) de son patriarche défunt ${ }^{9}$.

Dans le contexte de la diaspora - et plus précisément peut-être du syncrétisme mystique californien - le javânmardisme interconfessionnel des Iraniens tend à évoluer vers une espèce d'écologie spirituelle qui se réclame alternativement des grandes figures de la culture persane comme les grands poètes Hafez et Roumi, d'une vision rationaliste du monde, du probabilisme de la physique quantique, d'un dynamisme ésotérique, de la vertu de la prière - et dont l'enseignement initiatique ou pseudo initiatique (payant ou gracieux) est dispensé en persan tantôt dans l'enceinte de UCLA ${ }^{10}$, tantôt dans le cadre de la section iranienne du Rotary Club ou dans celui d'un centre islamique, par des personnalités telles que l'architecte Sahabi, la doctoresse en métaphysique Madame Sabahat, et le Docteur Mike Mirahmadi, devant des assistances pluriconfessionnelles et éduquées qui boivent leurs paroles avec ravissement. L'un des thèmes majeurs de ce soufisme new age est le souci de purification qui permet à l'individu de s'approprier sa propre existence à travers la maîtrise du monde, si ce n'est l'inverse. D'une certaine manière il participe de cette autoréflexivité du soi qui est le propre de la condition postmoderne, singulièrement en Amérique du Nord.

L'irréversibilité de l'expatriation et du fait diasporique devient évidente lorsque l'on considère la réorientation des pratiques évergétiques et funéraires. L'affectation des dons recueillis au sein de la communauté à des œuvres localisées en Iran se heurte de plus en plus aux réticences d'une partie de la diaspora qui ne voit pas l'intérêt de conforter le régime, redoute le détournement de ces aides et surtout souhaite en faire bénéficier ses propres nécessiteux (par exemple ces jeunes filles que l'on fait venir d'Iran à des fins de prostitution plus ou moins déguisée et qui du jour au lendemain se retrouvent à la rue, ou encore ces gens tout à fait honorables qui n'osent pas demander l'argent dont ils ont grand besoin). C'est ainsi que Madame Bahador Zadeh ${ }^{11}$, surnommée la Mère Teresa iranienne, a entrepris la construction d'une maison de retraite dans Orange County (l'un des principaux districts de Californie) n'hésitant pas à faire preuve d'un certain sens des affaires en revendant trois millions de dollars le terrain dont on lui avait fait don à cet effet pour en trouver un mieux situé, ni à mobiliser les

9. Ce que montrent par exemple les faire-part publiés dans Iranian Jezvish News, 2000.

10. Soixante dollars pour trois séances organisées sur trois mois par un architecte, le Docteur Sahabi, qui vit au Canada, mais qui est accueilli par sa famille lors de ses passages réguliers à Los Angeles et à Irvine.

11. L'une des membres du comité directif de Kahrizak, une maison de retraite (l'une des plus réputées) situcé au sud de Téhéran. 
bonnes volontés du réseau ethno-national pour offrir des services à des membres du troisième âge installés en Californie ${ }^{12}$ (et peut-être plus en butte à l'ennui qu'à de réels problèmes financiers). Ainsi, le temps de la vieillesse est maintenant destiné à être vécu sur la Côte Ouest, et les éventuels voyages en Iran se pensent non plus comme un retour, mais comme des moments de villégiature ou de ressourcement. Pareillement, le Docteur Namazikhah, quand on l'interroge sur l'usage qui sera fait des bâtiments qu'il construit à tour de bras, a une double réponse : d'une part, il attend avec une impatience rigolarde le jour $\mathrm{J}$ où l'ensemble de la communauté iranienne de Californie pliera bagage; de l'autre, il transformera alors son centre (baptisé centre de l'IMAN) en un musée de la diaspora dissoute. Cette répartie, très américaine dans cette préoccupation ironique de muséification, rappelle également combien les pratiques évergétiques, si caractéristiques de l'ethos économique iranien, se sont en définitive enrichies d'éléments empruntés à la société d'accueil. Les différentes formes d'adhésion à l'IMAN - simple membre, membres d'argent, d'or ou de platine - renvoient à des privilèges différenciés (détention d'une carte, octroi d'une chaise personnelle dans l'amphithéâtre, pose d'une plaque nominative sur le mur mémorial, voire à l'entrée de l'une des pièces du complexe). La terre d'opportunité est aussi la scène de l'émulation et de la distinction, que l'on soit ou non dans un cadre caritatif. Comme nous l'avons déjà dit, les évergètes irano-californiens recourent aux techniques les plus classiques du fund raising caritatif, telles que la mise aux enchères d'un tableau représentant l'imam Ali ou la compétition entre donateurs, l'un de ceux-ci pouvant annoncer qu'il "fera match" (match mikone $($ ), c'est-à-dire qu'il doublera automatiquement de sa propre poche la mise rassemblée par les autres participants.

Acceptation ultime de l'expatriation, des cimetières musulmans se proposent désormais d'abriter la dépouille des défunts iraniens. La commémoration des morts dans leurs allées est devenue une scène relativement familière, photos souvenirs à l'appui, notamment lors d'Achoura (l'un des jours de deuil dans le calendrier religieux chiite, qui célèbre le martyre de l'imam Hossein à la bataille de Kerbela). La pérennisation de la présence iranienne sur la Côte Ouest devrait atteindre son apogée avec l'utopie de Mahmood Moosavie, "martyr vivant " de la guerre avec l'Irak, ayant bénéficié de traitements hospitaliers aux Etats-Unis grâce à l'aide de la Fondation des déshérités, et qui s'y est finalement

12. L'un de mes interlocuteurs, actionnaire de l'Express Shuttle, m'a fait un jour part, non sans un certain enthousiasme, de sa contribution malgré lui aux ceuvres évergétiques. L'appel était venu d'un collègue, membre du réseau Kahrizak en Californie, qui sollicita, un beau matin, la mise à la disposition gracieuse d'un véhicule à un groupe de femmes âgées iraniennes, pour une journée, et ce afin de leur fournir une distraction nécessaire à leur équilibre. Madame Ashraf Bahador Zadeh faisait partie du groupe. 
installé. Convaincu que $l^{\prime}$ «Amérique a soif d'islam», il travaille à l'édification d'une cité islamique à une centaine de kilomètres de Los Angeles, qui comporterait logements, écoles, supermarchés, banques, cimetières, hôpitaux, et bien sûr mosquée. Il ne voit de solution que dans la formation en Amérique même, d'un nouveau clergé chiite, les clercs venus d'Iran étant voués soit à l'impuissance soit à la déviance.

Tout indique donc que cette communauté d'exilés, d'émigrés, d'expatriés est en passe de se constituer en véritable colonie, à l'interface d'une identité culturelle (voire d'une citoyenneté) américaine et d'une identification à l'Iran. Forte de son capital culturel et financier initial - son apport de capitaux aux Etats-Unis se serait élevé à trente ou quarante milliards de dollars au lendemain de la Révolution ${ }^{13}$-, elle jouit désormais d'un positionnement économique autonome que conforte son taux élevé de self employement ${ }^{14}$. Sa prospérité doit certes beaucoup aux flux de relations avec la mère patrie, mais elle ne $s^{\prime} y$ réduit pas. D'une part, les entrepreneurs irano-californiens accumulent à partir du marché américain, au-delà de sa composante ethno-nationale iranienne, et éventuellement en partenariat avec d'autres communautés (par exemple avec les milieux d'affaires chinois). Ils prospèrent dans les professions libérales, mais également dans différents services tels que les transports urbains, le design, l'informatique, le BTP, l'épicerie de gros, la joaillerie, la vente de tapis, le textile, ou encore dans l'immobilier. D'autre part, les opérateurs irano-californiens sont insérés dans un réseau de relations mondiales qui englobent, outre l'Iran, certaines places fortes de la diaspora, telles que Dubaï, la Turquie, Chypre, Tokyo, le Canada, Londres ou des grandes villes allemandes. Il est par exemple éloquent que, faute de liaisons aériennes directes entre les Etats-Unis et l'Iran pour cause d'embargo, les agences de Westwood proposent des billets pour Téhéran via Londres, Francfort ou Tokyo, ou encore que des petites annonces de la presse communautaire vantent les services de juristes capables d'obtenir une Green Card pour des Iraniens résidant au Japon ou à Dubaï.

En d'autres termes, l'économie politique de la colonie irano-californienne est bel et bien "globale ». Elle tire sa force non pas seulement d'une relation privilégiée avec l'Iran ou du seul marché local, mais des différentiels constitutifs de l'économie internationale. Ainsi, elle sait exploiter les atouts de la City, de la zone franche de Dubaï ou de l'accord de libre-échange nordaméricain (ALENA). Telle personnalité du monde évergétique partira à Londres avec les recettes des ventes de charité avant de gagner l'Iran; telle autre achètera en Allemagne les fauteuils roulants destinés aux mutilés de la

13. Ansari (M.), The Making of the Iranian Community in America, New York, Pardis Press, 1992, p. 127.

14. Kelley (R.) et al., Irangeles, Iranian in Los Angeles, Berkeley - Los Angeles, University of California Press, 1993, p. 74. 
guerre irano-irakienne. Dubaï est la principale place où se convertissent en dollars les capitaux quittant l'Iran pour, entre autres, la Californie. De ce point de vue le Mexique mérite une mention particulière. Il est le premier point d'entrée des produits de contrebande (tapis, antiquités), des migrants clandestins et des capitaux non déclarés qui ont pour destination la communauté. En outre il offre aux entrepreneurs irano-californiens, comme aux autres milieux d'affaires, des opportunités appréciées de délocalisation industrielle ou commerciale en raison des bas salaires et des terrains moins coûteux qu'ils y trouvent ${ }^{15}$. L'intensité de ces liens économiques entre le Mexique et les Iraniens de Californie a finalement donné naissance à un tissu humain transnational. Les "mariages temporaires" (pour reprendre une expression ironique souvent entendue) entre hommes iraniens et femmes mexicaines semblent fréquents, notamment dans le milieu des grossistes de Downtown, et on parle désormais d'enfants métis. Par ailleurs les entrepreneurs ou les familles iraniens ont évidemment des employé(e)s mexicain(e)s dont on dit qu'ils sont les "Afghans de la Californie » et qui parlent le persan comme seconde langue - une histoire, réelle ou apocryphe, veut qu'un invité s'étonne d'entendre son ami restaurateur parler en persan avec son serveur et se voit répondre: "Tais-toi, il croit que c'est de l'anglais. "

Le mode d'organisation de cette économie irano-californienne est ce que l'on pourrait nommer le "néo-vaqf": le vaqf islamique évolue dans un environnement culturel, juridique et fiscal américain, revêt une dimension intercontinentale, est dirigé par des diplômés de l'enseignement supérieur ${ }^{16}$ (lesquels font généralement œuvre évergétique en famille). De façon assez classique, l'institution sociale du néo-vaqf remplit toute une série de fonctions autres que caritatives ou pieuses. Il permet à l'opérateur iranocalifornien de légitimer sa relation avec l'Iran aux yeux d'une communauté toujours soucieuse de ne pas consolider la République islamique, mais aussi à ceux des autorités américaines attachées à leur politique de sanctions à l'encontre de Téhéran. Il procure à l'évergète-homme d'affaires une autonomie vis-à-vis de l'Etat iranien. Il offre des emplois aux membres de sa famille. Il est source d'exemption fiscale. Il mobilise des dons qui, certes, serviront à financer la cause annoncée, mais dont la gestion fournit une trésorerie appréciable pour réaliser d'autres opérations. Il n'interdit pas d'engranger des profits au fur et à mesure que sont effectués les actes de

\footnotetext{
15. Le Nefuork of Iranian American Professionals of Orange County (NIPOC) a précisément organisé, en juillet 2000, un séminaire sur le thème des opportunités qu'offre le Mexique.

16. Le iaqf traditionnel de bazar se caractérisait par un évergétisme de notables commerçants, souvent âgés, non diplômés; il n’impliquait ni lés femmes ni même les enfants. Mais il est vrai que cet univers du don a connu ces dernières années des mutations en Iran même: il tend à s'institutionnaliser, les femmes y jouent un rôle croissant, les acteurs de l'économic informelle et notamment les contrebandiers contribuent à sa prospérité, il peut faciliter le blanchiment des capitaux illicites (cf. Adelkhah (F.), Etre moderne' 'n Iran, op. cit., chap. 3).
} 
bienfaisance. Il accroît le prestige social et le réseau des relations du bienfaiteur. A terme, il crée un capital d'influence et de reconnaissance en Iran même, y compris dans les provinces.

L'exemple de la Fondation de la science et de l'art (Bonyâd-e dânesh va honar) illustre bien cette polyvalence fonctionnelle et morale du néo-vaqf. Fondée en mars 1999 à Londres (Imperial College) et immatriculée comme œuvre charitable aussi bien en Grande-Bretagne (1077499) qu'aux Etats-Unis (134087316), la Fondation s'est donnée pour objectif l'«empowerment » de la jeunesse du monde en développement à l'âge de l'information, notamment en améliorant son accès à la technologie de l'informatique et à l'internet. Elle a financé l'achat d'une dizaine d'ordinateurs par école bénéficiaire et la mise en réseau de dix-huit établissements scolaires en $\operatorname{Iran}^{17}$ (dont dix localisés à Téhéran et les huit autres à Rey, Varamin, Eslamshahr et Ispahan). Elle draine ses ressources à travers un réseau de local support groups, notamment à Atlanta, Boston, New York, San Francisco, Washington DC et Los Angeles. Quel que soit le montant des dons recueillis, l'essentiel est sans doute ailleurs. Le créateur de la Fondation, Abbas Edalat, professeur de mathématiques et d'informatique à Imperial College, est devenu par ce biais l'interlocuteur et le correspondant de diverses institutions scolaires et universitaires iraniennes, en particulier la très renommée Sanati Sharif de Téhéran, et ce en partenariat avec diverses organisations économiques, dont la firme automobile Iran Khodro. Du rôle de donateur à celui $d^{\prime}$ intermédiaire et de prestataire, il n'y a qu'un pas dont on peut raisonnablement penser qu'il sera un jour franchi. L'Iran représente un marché informatique de 65 millions de consommateurs où tout reste à faire et qui est potentiellement d'autant plus lucratif que la contrebande, le piratage, la régulation oligopolistique et la pénurie de devises prévalent même si, dans la pratique, ces avantages comparatifs peuvent s'avérer être de redoutables obstacles ${ }^{18}$. En outre, la relation à l'Iran n'est pas exclusive et, le relais dubaïote aidant, le Pakistan et l'Inde ouvrent d'autres perspectives.

La trajectoire personnelle de Pierre Omidyar, véritable javânmard de l'internet, n'est pas si différente. Né en France où il a vécu jusqu'à l'âge de six ans, il a fondé en 1995 eBay, un site d'enchères entre particuliers qui s'est imposé comme l'un des quatre grands noms de la "toile ", l'une des rares

17. Bilan d'activité exposé lors de la réunion organisée, à la demande de la Fondation ellemême, à l'IMAN le 10 juin 2000 . Le nombre des participants à cette rencontre fut limité, tandis qu'une autre réunion organisée à Orange County semble avoir accueilli une centaine de personnes. Dans les deux cas, le droit d'entrée s'élevait à cinquante dollars.

18. L'histoire racontée par Abbas Edalat, lors de son intervention à l'IMAN, d'un nombre somme toute limité d'ordinateurs d'occasion, réhabilités, expédiés en Iran et bloqués à la douane de Téhéran, est à ce propos assez parlante. Il a fallu plus de neuf mois et l'intervention des représentants de l'Iran à l'Unesco et à l'ONU, en plus de celle du directeur du Haut conseil de l'informatique, pour mettre fin au stockage de ces appareils qui, vraisemblablement, avaient comme seul défaut leur provenance : l'Europe, et non Dubaï, comme cela se fait d'habitude ! 
entreprises de la « nouvelle économie " qui a été d'emblée rentable - non, il est vrai, sans commercialiser quelques produits illégaux (tels que des armes, des organes humains ou de la drogue), un faux Rembranut et des ovules de mannequins... « Le site est créé tous les jours par les clients. Ce sont eux qui exercent le contrôle le plus efficace. Quand quelque chose leur paraît douteux, ils prennent contact avec nous, on étudie la situation et on retire l'objet» se justifie Pierre Omidyar qui, décidément très global, boude désormais les fastes de la Silicon Valley et réside à cheval sur le Nevada et la France, où il a lancé une version de son site ${ }^{19}$.

Du point de vue de la globalisation de l' "iranité ", l'islam, religion monothéiste, est universaliste : il permet à ses croyants de transcender, sans pour autant les renier, leurs identifications particulières de type régionaliste, ethnique, nationale ou même confessionnel. Etre musulman, ce n'est pas cesser d'être iranien, mais c'est sûrement dépasser une conception étriquée de l' "iranité », cette "fétichisation "si caractéristique de la communauté irano-californienne. Il est remarquable que, par cercles concentriques, les Iraniens de Californie musulmans développent, de pair avec une solidarité communautaire ethno-nationale, une sociabilité chiite transnationale et, audelà, un sentiment d'appartenance à un ensemble islamique qui englobe évidemment leurs coreligionnaires sunnites. De même que, dans les autres implantations de la diaspora, les lieux de pèlerinage et de dévotion sont souvent fréquentés simultanément par les fidèles des deux confessions - par exemple dans le Golfe, en Syrie ou dans le Baloutchistan -, les Iraniens chiites, avant que ne soient ouverts leurs propres centres, ont longtemps contribué aux activités de la grande mosquée sunnite de Vermont, à Los Angeles, dirigée par les frères Hassan et Maher Hathoot, deux Egyptiens, mais où les Pakistanais et les Afro-Américains musulmans se retrouvent nombreux. Ceux-ci restent d'ailleurs attachés à cette mosquée, continuant de recevoir ses publications ou d'être en partie tributaires de son magistère, par exemple quand il s'agit de préciser le calendrier lunaire (heures du lever et du coucher du soleil, cycle de la lune, décisifs notamment au moment du Ramadan). Bien sûr, tout cela ne va pas sans tensions au sein de l'Ummat (la communauté des croyants), bien que ces tensions n'aient jamais atteint l'acuité des affrontements entre sunnites et chiites à Nashville (Tennessee) ou à Ohio (Dayton). Andalibian Tehrani, du Islamic Service Center, tient des propos très durs à l'encontre des sunnites qui ont " cassé le dos " de Zahra, la fille du Prophète, ou des chiites enclins à se " saoudiser »; et un Attar, clerc irakien ayant fait ses études à Qom, considère que la République islamique est à l'origine de l'isolement et du retard de l'institution religieuse chiite au regard des transformations du monde.

19. Mauriac (L.), “ Ebay version France, gare aux enchères ”, Lihiration, 5 octobre 2000. 
Quoi qu'il en soit, l'islam demeure un accès à l'universel, et ce d'autant plus qu'il se veut désormais, sans complexe et " américain " - comme le dit dans un de ses discours, non sans une certaine ironie, le président de la Fondation Assadiq, Ali Ghazvini ${ }^{20}$. Sa langue véhiculaire est de plus en plus l'anglais et son souci est de devenir une composante du multiculturalisme aujourd'hui constitutif des Etats-Unis. Si les gays sont parvenus en une vingtaine d'années à passer du statut de déviance sexuelle honnie à celui d'une communauté ayant droit au respect " politiquement correct », déclare en substance Mike Mirahmadi du Islamic Center of Beverly Hills, pourquoi les musulmans n'arriveraient-ils pas a fortiori, à leur tour, à éliminer les préjugés dont ils sont l'objet ? Cet " islam américain " porte volontiers cravate. Tel est par exemple le cas d'Ali Ghazvini, alors qu'il est l'aîné d'un clerc prestigieux et qu'il est lui-même un ancien fonctionnaire de la République islamique. De façon révélatrice, celui-ci insiste d'ailleurs sur le fait qu'il n'est pas en Californie en tant que représentant (ou pour le compte) de l'Etat iranien, mais bel et bien en tant que musulman nord-américain décidé à prendre sa part de cette "société avancée" (jâme'eh pishrafteh). Encore une fois il convient d'être nuancé. Le souci de vivre son islam à travers les techniques les plus modernes de communication et à travers des centres polyvalents qui n'ont plus grand-chose à voir avec les mosquées traditionnelles fait bon ménage avec un solide conservatisme social : le port de la cravate par les hommes n'est pas censé affranchir les femmes de celui du voile.

Il n'empêche que ce conservatisme ne mobilise pas que des vieilles barbes. $C^{\prime}$ est bien à travers les circuits et les centres caritatifs islamiques que les femmes participent à l'espace public, conformément à ce que l'on a pu par ailleurs observer aussi bien dans la République islamique que dans les institutions charitables chrétiennes occidentales; et les jeunes hommes, quand ils ne sont pas dans les boîtes de nuit, sont très présents dans les célébrations religieuses ou dans les fêtes culturelles. De ce point de vue, la capacité d'attraction de l'islam sur les forces vives de la communauté est indéniablement plus grande que celle des mouvements politiques, largement désertés par les jeunes générations. Il n'y a guère que les cabarets ou les soirées privées qui puissent lui faire ombrage, notamment chez les jeunes filles, décidément plus enclines à danser l'arabi, le bandari et le bâbâ karam qu'à participer à des réunions dévotes - sans doute parce que l'espace public reste pour elles chargé de morale, d'admonestations et d'interdictions, quand il est pour leurs frères une instance où « rouler les mécaniques ", débattre, organiser et exercer un leadership. Sans que cela soit très original à l'échelle de la société nord-américaine, un tel conservatisme mobilisateur, fort de ses ressources institutionnelles, de son système scolaire en pleine effervescence et de son propre calendrier rituel amovible et

20. Dans le discours officiel de la République, l'islam « américain » est censé s'opposer à l'islam authentique ou l'islam sans concession qui prévaudrait en Iran. 
flexible, participe de plain-pied au changement social en Amérique. Ainsi, il s'efforce à sa manière de recomposer la relation entre l'islam et la démocratie. Une revue comme Th' Minaret, publiée par la mosquée de Vermont, n'hésite pas ainsi à consacrer l'une de ses livraisons aux Civil Rights ; et les notables de la communauté se félicitent d'être reçus désormais annuellement, à l'instar des représentants des autres confessions, par le Congrès au mois de février, ou par le Président au mois de novembre, pour un petit-déjeuner à la Maison Blanche.

L'une des expressions de ce dynamisme de l'islam américain est justement sa dimension notabiliaire. Si la réislamisation de la colonie iranienne en Californie est en partie le fruit de l'arrivée d'une nouvelle vague d'immigrants, moins fortunée, ou des regroupements familiaux (au profit souvent des parents), elle est désormais conduite par des élites éduquées et prospères, telles que des médecins, des dentistes, des grossistes, des professeurs d'université, des lawuyers et, last but not léast, des clercs. Ici comme ailleurs, c'est une grossière erreur que de voir dans ce processus une manifestation de la pauvreté et de la marginalité. Avant d'être le cri des damnés de la terre, l'islam peut être la griffe de la réussite sociale; le favoriser par le biais des néo-iaqf est susceptible de devenir source d'enrichissement. Le contrôle de ces nouveaux modes de bienfaisance est d'ailleurs l'objet de conflits entre les dirigeants de la communauté. La multiplication des centres religieux s'explique en partie de la sorte, comme l'a illustré la scission de l'IMAN à l'initiative de Dastmaltchi et Abedi. Ces derniers ne se satisfaisaient plus de la perpétuation du leadership du centre et auraient souhaité une direction tournante. En tant que tel, l'islam n'est pas seulement un vecteur de solidarité et d'identité mais aussi une machine à fabriquer - ou en tout cas à consacrer - de l'inégalité sociale. La manière dont le tirage au sort, lors de la célébration de la Fête des mères, peut bénéficier aux grandes familles dirigeant le centre de l'IMAN pour la distribution des cadeaux offerts par les bienfaiteurs n'est sans doute pas que le fruit du hasard... Enfin et surtout, à Los Angeles comme à Dubaï et à Tokyo, le marché cambiaire a représenté depuis 1980 une ressource notabiliaire formidable pour les émigrés, compte tenu de la faiblesse et de la dépréciation continue du rial des années 1980 jusqu'à 2001. La rémunération et les gains en devises fortes - par exemple en dollars, en yens, en livres sterling ou en euros - constituent un avantage formidable par rapport à des gens qui touchent leurs revenus en rials. Ils confèrent aux stratégies de la bienfaisance libellées dans ces monnaies une efficacité redoutable : l'argent envoyé au pays, les investissements réalisés dans les "petites patries ", etc., ont un pouvoir social très élevé, même si celui-ci a tendu à s'effriter ces dernières années en raison de l'augmentation du coût de la vie en Iran et surtout de la flambée du marché immobilier dans les principales villes du pays. 


\section{Le retour du notable}

Comme tout émigré, le notable est "parti sans quitter ": il reste attaché à son pays d'origine et à sa " petite patrie " par la mémoire, les liens familiaux, les échanges matériels et culturels ${ }^{21}$. L'on peut même dire qu'il a plus que d'autres les moyens de "rester", dans la mesure où il dispose de plus grandes ressources pour rapatrier des devises financières (ou des ressources de tout ordre) et pour faire acte d'évergétisme transnational - par exemple en donnant à des œuvres religieuses ou en prenant en charge des célébrations familiales. Mais cette projection du notable dans sa société de départ comporte ses particularités et ses distanciations propres. L'expérience sociale de l'expatriation va fréquemment de pair avec un certain conservatisme culturel qui procède par invention de la tradition. L'Iran que l'on célèbre et dont on a la nostalgie devient vite un artefact.

Lorsqu'il se réinsère dans sa société d'origine le notable expatrié se trouve facilement, de ce fait, en décalage par rapport à celle-ci. Il restera fidèle à des modes d'organisation et de hiérarchie sociale ou à des pratiques culturelles surannés, lesquels irriteront d'autant plus qu'ils sont en grande partie fantasmatiques. Voudra-t-il reconstituer l'harmonie et la solidarité de la famille élargie qu'il se heurtera à la dure réalité des conflits entre ses membres et ses branches ou au désir d'autonomie des jeunes générations. Voudra-t-il aménager son intérieur en style "traditionnel » (sonnati) qu'il déconcertera ses parents ou ses proches, adeptes du style « aristocratique » (ashrâfi) descendant de Louis XV, via le faubourg Saint-Antoine et les Galeries Barbès. A lui seul, ce chassé croisé résume d'ailleurs l'ambivalence de la relation notabiliaire dans l'expatriation: l'émigré qui réussit rêve d'authenticité culturelle, alors que le critère local de distinction sociale est un style emprunté à l'étranger. Et c'est bien dans ce type d'ambivalence que se noue le rapport notabiliaire. L'évergète n'est pas seulement le produit de sa propre stratégie d'éminence sociale ou d'aspiration éthique. Il est aussi (au moins en partie) créé malgré lui, et à son insu, par l'attente et les ruses de son entourage qui s'ingénie à se constituer en clientèle. L'émigré légitime son expatriation par l'aide qu'il apporte à sa famille ou à sa " petite patrie ". Mais cette assistance peut se retourner contre lui comme un piège, en raison de l'inflation des exigences des donataires. L'équilibre financier, voire psychologique, du donateur peut s'en trouver compromis s'il ne parvient pas à faire face. Lorsqu'il commence à rappeler que « lui aussi il existe», le voile des sentiments et de la solidarité se déchire et les conflits familiaux s'exacerbent. Dans certains cas limite, le notable expatrié est soumis à de véritables pratiques d'extorsion, chantage affectif à l'appui, voire à de véritables escroqueries, à l'instar de ce qui est arrivé à la chanteuse Googoosh et dont il a été question plus haut. Les procès sont au demeurant

21. Adelkhah (F.), « Partir sans quitter, quitter sans partir », Critique internationale, 19, 2003. 
nombreux entre émigrés et parents restés au pays. La condition expatriée n'est pas seulement une ressource notabiliaire, mais aussi un danger: l'éloignement rend plus difficile le contrôle de la clientèle à qui l'on dispense ses bienfaits et celui de l'utilisation des fonds que l'on alloue.

Par ailleurs, la stratégie du notable expatrié s'inscrit aujourd'hui dans un contexte particulier, celui de la libéralisation économique, qui accentue ses incertitudes et son ambivalence. Les investissements évergétiques que prodigue $l^{\prime}$ « homme de bien » de la diaspora sont assez spectaculaires. Ils ont notamment transformé le paysage social des provinces déshéritées du Sud de l'Iran ou de certaines petites villes d'autres régions en multipliant les équipements collectifs dans le domaine des transports, de l'éducation ou de la santé. Des villes comme Lar, Lamerd, Grash, Evaz, etc., se voient ainsi dotées d'aéroports internationaux ou d'hôpitaux ultramodernes bien qu'elles ne comptent que quelques dizaines de milliers d'habitants. En outre, la voie publique porte la marque de ces stratégies personnelles : les rues, les châteaux d'eau, les barrages, etc., seront baptisés des noms des " hommes de bien " qui les auront financés, plutôt que ceux des martyrs ou des dignitaires de la République islamique. Se posent alors le problème épineux de la renégociation des rapports entre la sphère privée et la sphère publique. L'appropriation symbolique de l'espace public par des évergètes est un sujet de jalousie et de compétition entre ceux-ci ou leurs familles; elle est en creux un facteur de hiérarchisation, de marginalisation ou d'exclusion sociales, puisque toutes les familles n'ont pas la possibilité de voir fleurir leur réputation au carrefour de la ville. Il s'ensuit des frustrations, des insinuations et des accusations plus ou moins explicites : d'où vient l'argent, sinon du vol, de la contrebande ou du trafic de drogue?

En outre, construire est une chose, mais entretenir en est une autre. Sitôt reconnue sa munificence, le notable cherchera à en faire porter le coût par l'Etat ou par les collectivités locales. Pour en donner un exemple, la construction de l'aéroport de Lar, d'où partent deux vols hebdomadaires directs pour Dubaï et qui a été inauguré par le président Hachemi Rafsandjani, a été financé par les commerçants du cru, mais sa gestion et son entretien ont été attribués au domaine public, sans que l'Etat s'interroge trop longuement sur la nature des capitaux mobilisés, ni sur les modalités de leur transfert des Emirats Arabes Unis à l'Iran. La même remarque peut être formulée à propos de l'hôpital ultramoderne et de l'université de cette même ville, en cours d'édification, bien que, dans ces deux cas, le statut du ‘’aqf, confié à un administrateur, semble avoir été préféré. L'équivoque de ces relations entre le public et le privé - ou entre l'éthique de la charité et l'esprit de lucre - ne va pas sans de multiples conflits d'intérêts. Les contentieux juridiques liés à la gestion des roaff sont passés de 157 en 1992 à 
599 en 1997 dans la seule province de Fars 22 . Ainsi, feu Mohammad Namazi avait construit le réseau d'adduction d'eau de Shiraz dans les années 1950 en précisant que les profits de sa gestion devaient aller au financement de l'hôpital qu'il avait édifié au même moment. Mais, si l'on en croit le président de l'Organisation des biens de mainmorte, le gestionnaire actuel de la compagnie des eaux (qui n'est rien moins que le ministère de l'Energie) ne respecte pas la volonté du mécène et omet d'en reverser les bénéfices à l'établissement hospitalier. Dans un autre genre, des évergètes de Grash n'ont pas hésité à édifier une mosquée, la plus grande de Shiraz, sous les fenêtres du superbe hôtel touristique que leurs éternels rivaux lari étaient sur le point d'ouvrir, afin que le muezzin en importune la clientèle !

Ces conflits aidant, un débat a lieu actuellement quant au statut juridique du vaqf. Celui-ci doit-il être considéré comme une propriété individuelle (celle de son fondateur, dont le vœu est inaliénable, et de ses héritiers, voire des bénéficiaires) ou comme une personne morale (qui serait somme toute similaire à une fondation occidentale, sinon à une société anonyme) ? Pour un juriste comme Nasser Katoozian, il conviendrait d'assouplir le cadre légal du vaqf, dans la mesure où sa vocation initiale peut devenir obsolète compte tenu de l'évolution de la société (est-il encore bien utile de racheter des cruches à des servantes qui les auraient cassées ?) et de permettre une gestion économiquement rationnelle de son capital en restant fidèle à l'esprit, plutôt qu'à la lettre, de l'intention originelle du fondateur. S'autorisant de l'avis théologique de plusieurs "sources d'imitation" (mardja'), il propose d'autonomiser le don par rapport au donateur, de ne voir dans le vaqf que l'établissement d'une relation à Dieu, de le dépouiller de toute raison sociale trop rigide et de le gérer de la façon la plus rentable qui soit du point de vue des intérêts de la communauté des croyants ${ }^{23}$. Mais l'un des enjeux, à terme, de cette réflexion implique l'assujettissement de l'évergétisme (et donc de la stratégie notabiliaire) à l'impôt. Jusqu'à présent, la pression fiscale effective a été très faible en Iran. Néanmoins les réformes de libéralisation économique rendent à peu près inévitable l'intensification du prélèvement des taxes. Même les puissantes fondations liées au régime n'y échappent plus, au moins en théorie. Il est clair que les biens de mainmorte se verront touchés d'une manière ou d'une autre, ce qui ne dissuadera naturellement pas les "hommes de biens" de s'affranchir de cette réglementation. Sans doute ceux d'entre eux qui sont expatriés y parviendront-ils plus facilement, ne serait-ce que parce que le régime cherche à les faire revenir et à leur faire jouer le rôle d'investisseurs

22. Riahi (N.), Vaqf, Ravabet-e omoumi-ye edareh-e koll-e oqâf va omouor-e kheiriyeh-e fârs, Safar, $1419 / 1999$.

23. Katoozian (N.), "Development of the Institution of Endowment and its Future Prospects: A Comparative and Historical Study ", An Anthology of Iranian Studies, 6, 2002. 
privilégiés qu'ont tenu les "Chinois d'outre-mer" vis-à-vis de la Chine populaire.

Il va sans dire que ce climat de libéralisation économique et de retrouvailles entre la République islamique et la diaspora, que le séisme de Bam semble avoir consacrées, est propice à toute sorte de dérives affairistes. Dès les années 1990, Jean-François Bayart parlait ironiquement de la "République des initiés " pour désigner le "chevauchement "systématique des positions de pouvoir et des positions d'accumulation ${ }^{24}$. L'ouverture croissante du pays sur l'extérieur, l'exacerbation de la lutte factionnelle entre "réformateurs" et "conservateurs » ou les privatisations en trompe-l'œil ont accentué ce glissement - et, simultanément, les appels à la répression de la corruption et de la contrebande. La vie politique a ainsi été émaillée de diverses affaires, telles que celle de la municipalité de Téhéran en 1997199825. Autant que de moralité publique, il s'agissait bien sûr de conflits de pouvoir. L'intérêt de ces scandales est entre autres de faire apparaître l'économie politique du régime et plus spécialement, pour ce qui nous concerne, de la notabilité. En ces temps de libéralisation et de privatisation, les évergètes doivent nager dans des eaux troubles.

De ce point de vue, l'affaire Jazaeri a été particulièrement éloquente. Ce jeune homme, âgé de 29 ans, a été arrêté le 15 novembre 2001 dans la cabine - de première classe, insisteront lourdement les médias - d'un avion en partance pour Dubaï, en compagnie de son épouse et de son beau-père. Ancien étudiant en dentisterie, fort de ses relations avec l'ayatollah Moghtadai, ami du fils de celui-ci, il s'est livré à des activités d'intermédiation à l'exportation, dans le cadre réglementaire qui imposait aux exportateurs de signer des accords de devises (peyman-e arzi) par lesquels ils s'engageaient, transaction par transaction, à faire rentrer, via la banque centrale, un montant précis de devises (ou sa contrepartie en produits importés) en accord avec les règlements. Les opérateurs s'exposaient à d'éventuels déboires s'ils ne parvenaient pas à réaliser la vente espérée ou si les cours sur le marché international baissaient par rapport à leurs prévisions. Ils devaient alors s'acquitter de leurs obligations bien qu'ils n'eussent pas engrangé le profit escompté, quitte à acheter sur le marché libre, au prix fort, les dollars qu'ils devaient revendre à la banque centrale $^{26}$. Jazaeri prenait à sa charge ce risque en en négociant les termes avec l'exportateur. Dans le même temps, sa position lui permettait d'investir à Dubaï et au Yémen et d'obtenir des crédits bancaires dans les pays arabes

24. Bayart (J.-F.), "Entre "dirigistes et "libéraux" : la République islamique ", in Adelkhah (F.), Bayart (J.-F.), Roy (O.), Thermidor ('n Iran, Bruxelles, Editions Complexe, 1993.

25. Adelkhah (F.), "Le maire, le ministre, le clerc et le juge. Le judiciaire et la formation du politique en République islamique d'Iran ", in Briquet (J.-L.), Garraud (P.), dir., Juger la politique'. Entriprises et entreprene'ur: iritiques de la politiqui', Rennes, Presses universitaires de Rennes, 2002. 26. Les procédures d'exportation ont été libéralisées dépuis. 
du Golfe en bénéficiant de la garantie de la Société de promotion des exportations (une société publique). Grâce à sa trésorerie, à sa cinquantaine de sociétés, à ses 142 comptes en banque et à ses gains, cet « islamo-golden boy " s'est posé en bienfaiteur du clergé, de l'université et du Parlement. Il avait d'ailleurs été auditionné par la commission économique de celui-ci à plusieurs reprises. Une soixantaine de députés, dont Mehdi Karroubi, le président de l'Assemblée, avait joui de ses largesses : téléphones portables, voitures, maisons, voyages à Dubaï et, sans doute, financement de campagnes électorales. Mais Jazaeri avait également beaucoup aidé des universitaires, des chercheurs, des clercs, sans même parler des œuvres sociales qu'il avait créées dans le Khousistan, sa région d'origine, ou des ressources financières qu'il avait distribuées dans cette même région. Accusé d'abus de biens sociaux et de trafic d'influence, il a bâti sa défense devant le tribunal sur le respect de ses engagements, sur la conformité de son action aux orientations économiques du guide de la Révolution, sur le caractère évergétique de sa générosité et sur la pureté de ses intentions. L'affaire a été étouffée, dans la confusion, après la quatrième audience publique de son procès. Mais l'interrogation demeure : Jazaeri était-il un vulgaire escroc? L'homme de paille trop bavard d'une partie de la classe politique ? Son bouc émissaire ? Un génie des affaires tel que l'Iran en manque cruellement ? Ou un jeune homme prodigue et ambitieux qui n'a pas su maîtriser une ascension notabiliaire trop rapide?

Le même type d'incertitudes se retrouve à propos des notables qui brillent dans la diaspora. Leur richesse est parfois impressionnante et leur munificence $d^{\prime}$ « homme de bien " est en conséquence. Dans plusieurs cas, on a quelque peine à penser que la vente de tapis, de vaisselle ou encore la dentisterie suffisent à les expliquer. Certains ont pu quitter l'Iran avec un capital important, notamment après la Révolution. D'autres ont fait fortune à l'étranger, mais sans doute en prospérant dans des activités d'intermédiaire (vâseteh) qui sont le ressort à la fois de l'économie et du javânmardi. Le tout est alors de savoir, d'une part, si ces activités sont licites et, d'autre part, si elles sont menées en partenariat avec des autorités, des institutions ou des entreprises publiques Elles peuvent être banalement commerciales, éventuellement sur le mode de l'informel, ou liées à des impératifs de sécurité nationale dès lors que l'Iran a été en guerre pendant huit ans et a été soumis à embargo et sanctions, ou franchement délictueuses, de la part d'opérateurs qui sont géographiquement voisins de l'une des principales régions productrices d'opium. La seule chose qui soit claire est que la libéralisation et l'internationalisation de l'économie iranienne est en train de transformer profondément les ressources traditionnelles du notable, sans pour autant remettre en cause son ethos. Le javânmardi continue de prospérer et de se recomposer à l'ombre des privatisations, et dans l'attente de l'adhésion de l'Iran à l'OMC. 